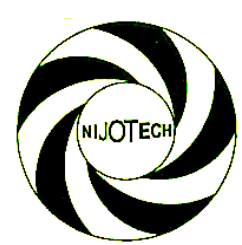

Nigerian Journal of Technology (NIJOTECH)

Vol. 39, No. 2, April 2020, pp. 351 - 362

Copyright@ Faculty of Engineering, University of Nigeria, Nsukka

Print ISSN: 0331-8443, Electronic ISSN: 2467-8821

www.nijotech.com

http://dx.doi.org/10.4314/njt.v39i2.5

\title{
DERIVATION AND OPTIMIZATION OF DEFLECTION EQUATIONS FOR TAPERED CANTILEVER BEAMS USING THE FINITE ELEMENT METHOD
}

\author{
M. M. Ufe ${ }^{1, *}$, S. N. Apebo ${ }^{2}$ and A. Y. Iorliam ${ }^{3}$

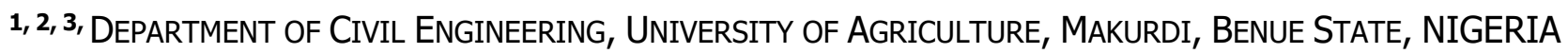 \\ E-mail addresses: 1 ufemoses@gmail.com, ${ }^{2}$ apebson@gmail.com, ${ }^{3}$ amosyalas2007@yahoo.com
}

\begin{abstract}
This study derived analytical solutions for the deflection of a rectangular cross sectional uniform/y tapered cantilever beam with varying configurations of width and breadth acting under an end point load. The deflection equations were derived using a numerical analysis method known as the finite element method. The verification of these analytical solutions was done by deterministic optimisation of the equations using the ModelCenter reliability analysis software and the Abaqus finite element modelling and optimisation software. The results obtained show that the best element type for the finite element analysis of a tapered cantilever beam acting under an end point load is the C3D20RH (A 20-node quadratic brick, hybrid element with linear pressure and reduced integration) beam element; it predicted an end displacement of $0.05035 \mathrm{~m}$ for the tapered width, constant height cantilever beam which was the closest value to the analytical optimum of 0.05352 $m$. The little difference in the deflection value accounted for the numerical error which is inevitably present in the analyses of structural systems. It is recommended that detailed and accurate numerical analysis be adopted in the design of complex structural systems in order to ascertain the degree of uncertainty in design.
\end{abstract}

Keywords: Deflection, Finite element method, deterministic optimisation, numerical error, cantilever beam.

\section{INTRODUCTION}

There are numerous analytical methods for finding deflection of beams such as the Double integration method, the Macaulay method and the moment area methods [1]. However, these methods are applicable to simple structural systems where there is simple loading and where the beam is prismatic and of uniform cross section. In real life situations where the beam may have a varying cross section, or subjected to multiple or complex loading; other methods such as the finite element method may have to be employed. This research work used the principle of virtual work and finite element theory to find the deflection equations for tapered cantilever beams of rectangular cross sectional areas acting under an end point load. The verification of these equations was done by deterministic optimisation in ModelCenter reliability analysis software and in Abaqus. Abaqus is a software application used for modelling and analysis of mechanical components [2]. It is used in finite element modelling and analysis.
Engineering designs aim at getting the best parameters that will reduce the cost of production and increase the performance. The process of doing this is known as optimisation [3]. Structural optimisation theory involves stating of structural engineering problems as mathematical programming problems. It is better than the conventional structural design methods because of its efficiency and time-saving ability. The theory and application of structural optimisation have increased greatly over the years basically as a result of the implementation of high computing and the finite element method in engineering [4]. In a conventional optimisation problem, the set of design parameters which are changed in order to get the optimum performance are known as the design variables while the mathematical model used for investigating the merits of the engineering performance is known as the objective function. The function dividing the design space between feasible and invalid region is known as the constraint. The constraints are divided into the

* Corresponding author, tel: +2348059368909 
inequality constraints and the equality constraints. The design variables are usually contained in the objective function and the constraints [5]. In mathematical terms, an optimisation problem is typically stated as follows;

$$
\begin{array}{lc}
\text { optimise } & f(x), \\
\text { subject to: } & \mathrm{g}_{i}(x) \leq 0, \quad i=1, \ldots, I, \\
h_{j}(x)=0, & j=1, \ldots J
\end{array}
$$

Where $x \in X$ is a set of design variables and $f(x)$ is the objective function. Equation 1(b) represents the set of inequality constraints and 1 (c) the set of equality constraints.

In a structural optimisation problem, the design variables could be the width or breadth of a reinforced concrete beam or the quantity of cement in a concrete mix and the objective function could be the weight of the reinforced concrete beam. In the theory of deterministic optimisation, the design variables are given exact values and the objective function and constraints are also assumed to be deterministic [6]. Based on the nature of design variables in engineering, structural optimisation problems are divided into sizing, shape and topology optimisation. Sizing optimisation involves the checking of structural dimensions that give desired performances at low cost; shape optimisation involves the checking of the design parameters that give the best geometrical properties defining basic structural shapes while topology and layout optimisation is the identification and location of vacuums or voids in continuous structures or the determination of the number of joints or connections in discrete structural systems [7].

There are several approaches utilised in the development of solutions to structural engineering problems. The finite element method is utilised when the structural system for analysis is too large to be handled by simple analytical methods. They are mostly used in the solution of continuums and involve developing solvable analytical solutions for discrete elements of the continuum [8]. The representation of structural engineering problems by simplified mathematical equations is as old as the origin of engineering itself. The characterisation of real structural systems by simple algebraic equations is known as mathematical modelling. The solution of these mathematical expressions gives the solution to various structural properties. The equations are solved by analytical and numerical methods [9]. The analytical solution methods are applied to systems which can be solved by simplified differential equations and they usually give accurate and exact solutions while Numerical methods are approximate methods. The Finite element method is a numerical solution method that is applied to large engineering systems (continuums) which cannot be solved accurately as a whole and therefore requires the continuum to be broken into small and discrete meshes. Each mesh contains a group of elements known as finite elements. The solution is then sorted at the nodes of the meshes where the elements meet, after which the nodal solutions are interpolated to give a global solution for the structural system [10].

There are different kinds of finite elements including the bar element, triangular, quadrilateral element, beam element, truss element, shell and plate elements. The best finite element type that gives the desired solution to the structural problem is usually adopted for mesh refinement analysis. This is also done to find out the mesh density that best approximates a structural solution. There are two types of mesh refinement known as $h$ and $p$ mesh refinement. The fundamental principle of finite element analysis is known as the interpolation theory. It is the principle used in combining the nodal vectors gotten from the discretised system into global vectors [11]. For a linear bar element (Figure 1), the polynomial function describing the interpolation scheme is as follows.

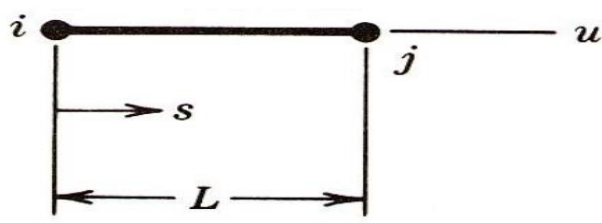

Figure 1: The linear bar element

$$
\emptyset=a_{1}+a_{2} s
$$

Where $a_{1}$ and $a_{2}$ are the nodal vectors and $\varnothing$ is the global vector.

The shape functions for a finite element describes how the global unknown vectors are interpolated from the known nodal vectors. The shape functions for the bar element are as follows.

$$
\left(N_{1}, N_{2}\right)=\left(\frac{1-s}{L}, \frac{s}{L}\right)
$$

Where $N_{1}$ is the shape function corresponding to node $i$ and $\mathrm{N}_{2}$ is the shape function corresponding to node 
$j ; s$ is the horizontal distance from node $i$ and $\mathrm{L}$ is the length of the bar element.

The algebraic equations emanating from the mathematical modelling of continuums are best solved by computer software applications as trying to solve them manually is very hectic and practically impossible [12]. The structural system to be analysed should be modelled and simulated with care since any modelling errors in the analysis may result in a wrong output from the software. Abaqus is one of the most useful software used in finite element analysis. It has a library of finite elements which are used in various types of structural analysis. Some types of elements, when used in a specific analysis, give a better approximation to the real solution than others. For example, it has been proven that the quadratic shell elements give a better approximation to the exact end displacement and stress in a twisted cantilever beam under the action of an in-plane and out-plane end loading than the triangular shell elements [13]. Some of the shell elements used in Abaqus and their description are as shown in Table 1 [14].

\section{DEVELOPMENT OF THE DEFLECTION EQUATIONS}

For a uniformly tapered cantilever beam with a rectangular cross-sectional area as shown in Figure 2, the displacement as a function of distance, $x$ from the fixed end of the cantilever beam subjected to a point load at the tip of the free end was found.

The cantilever beam has a tapered width $\mathrm{w}(\mathrm{x})$ and tapered height $\mathrm{h}(\mathrm{x})$. The development of the deflection equations for this structural system was done in stages. First, the height of the cantilever beam was kept constant and the width tapered and the formula for the end displacement developed, after which the width of the cantilever beam was kept constant and the height tapered and the end displacement formula found. The following sections present the derivation of the end displacement for the various configurations of the cantilever beam.

Table 1: Some commonly used shell elements

\begin{tabular}{ll}
\hline S3 & $\begin{array}{l}\text { A three-node triangular general purpose } \\
\text { shell, finite membrane strains. This is a } \\
\text { linear element }\end{array}$ \\
\hline STRI & $\begin{array}{l}\text { A six-node triangular thin shell, using five } \\
\text { degrees of freedom per node. This is a } \\
\text { quadratic element }\end{array}$ \\
\hline
\end{tabular}

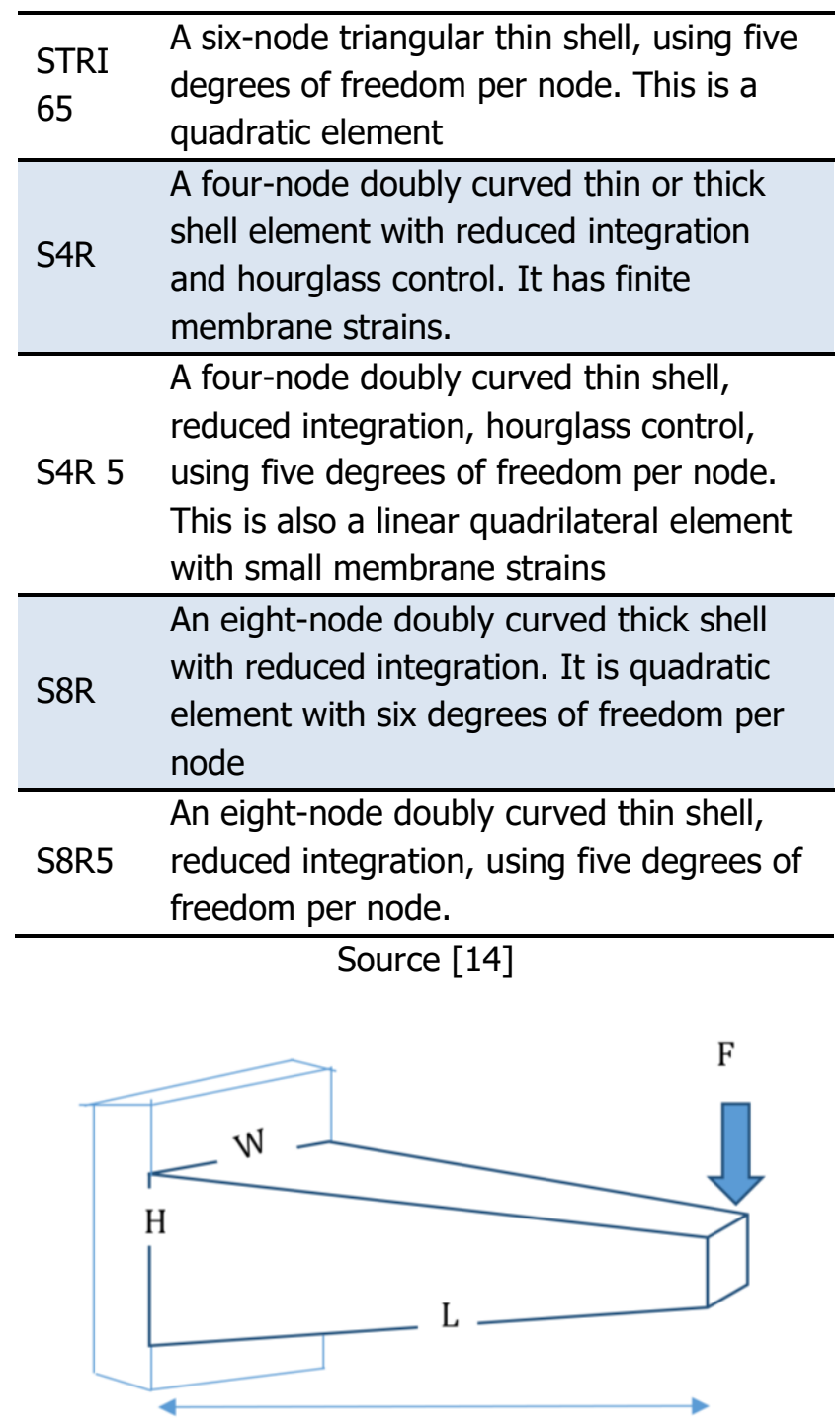

Figure 2: A tapered cantilever beam under an end point load

\section{CANTILEVER BEAM WITH A TAPERED WIDTH AND CONSTANT HEIGHT}

First, the tapered width of a constant height cantilever beam was taken as a function of only the width at the fixed end, $w_{1}$ of the cantilever without including the width at the free end, w2 as follows.

$$
w(x)=W\left(1-\frac{x}{a L}\right)
$$

Where $\frac{1}{a}=$ percentage taper of the beam; $w(x)$ is the width at a distance $x$ from the fixed end and $\mathrm{W}$ is the width at the fixed end of the cantilever beam.

By the principle of virtual work the strain energy, $U$ in the beam is as follows [15];

$$
U=W_{\text {bend }}-F \cdot y\left(L_{c}\right)
$$


Where, $W_{\text {bend }}=$ bending energy; $F$ is the point load at the free end and $y\left(L_{c}\right)$ is the deflection at the free end of the cantilever beam

$$
W_{\text {bend }}=\frac{1}{2} E \int_{0}^{L_{c}} I_{z}(x)\left[\frac{d^{2} y}{d x^{2}}\right]^{2} d x
$$

Where $\mathrm{E}$ is the elastic modulus of the beam and $I_{z}(x)$ is the moment of inertia at a distance $x$ from the fixed end of the cantilever beam

The assumed deflection curve of the beam and is approximated as follows;

$$
y(x)=C_{2} x^{2}+C_{3} x^{3}
$$

The moment of inertia of the beam of rectangular cross-sectional area is as follows;

$$
I_{z}(x)=\frac{w(x) H^{3}}{12}
$$

Therefore, the strain energy in the beam becomes;

$$
\begin{aligned}
& U=\frac{E}{2} \int_{0}^{L} \frac{w(x) H^{3}}{12}\left[\frac{d^{2} y}{d x^{2}}\right]^{2} d x-F\left(C_{2} \mathrm{~L}^{2}+C_{3} \mathrm{~L}^{3}\right) \\
& U=\frac{E W H^{3}}{24} \int_{0}^{L_{C}}\left(1-\frac{x}{a L}\right)\left[\frac{d^{2} y}{d x^{2}}\right]^{2} d x-F\left(C_{2} \mathrm{~L}^{2}+C_{3} \mathrm{~L}^{3}\right) \\
& U=\frac{E W H^{3}}{24} \int_{0}^{L_{c}}\left(1-\frac{x}{a L}\right)\left[2 C_{2}+6 C_{3} x\right]^{2} d x-F\left(C_{2} \mathrm{~L}^{2}+\right.
\end{aligned}
$$

By integrating equation (11) by parts, it follows that;

$$
\begin{aligned}
& U=\frac{E W H^{3}}{24}\left[-8 a C_{2}^{3}-\frac{16 C_{2}^{4}}{24 C_{3} L}+(a-1)\left(2 C_{2}+6 C_{3} L\right)^{3}+\right. \\
& \left.\frac{\left(2 C_{2}+6 C_{3} \mathrm{~L}\right)^{4}}{24 C_{3} L}\right]-F\left(C_{2} \mathrm{~L}^{2}+C_{3} \mathrm{~L}^{3}\right)
\end{aligned}
$$

By expanding the polynomials in Equation (12) and simplifying

$$
\begin{aligned}
& U=E W H^{3}\left[\left(\frac{4 a-3}{8 a}\right) \cdot C_{3}^{2} \mathrm{~L}^{3}+\left(\frac{3 a-2}{6 a}\right) C_{2} C_{3} L_{c}^{2}+\right. \\
& \left.\left(\frac{2 a-1}{12 a}\right) C_{2}^{2} L\right]-F\left(C_{2} \mathrm{~L}^{2}+C_{3} \mathrm{~L}^{3}\right)
\end{aligned}
$$

In order to find the values of $C_{2}$ and $C_{3}$ that bring the strain energy in equation (13) to zero, the partial derivatives of $U$ with respect to $C_{2}$ and $C_{3}$ will be set to zero

$\frac{\partial U}{\partial C_{2}}=\left[E W H^{3}\left(\frac{3 a-2}{6 a}\right) C_{3}-F\right] \mathrm{L}^{2}+E W H^{3}\left(\frac{2 a-1}{6 a}\right) C_{2} \mathrm{~L}=0$ $\frac{\partial U}{\partial C_{3}}=\left[E W H^{3}\left(\frac{4 a-3}{4 a}\right) C_{3}-F\right] \mathrm{L}^{3}+E W H^{3}\left(\frac{3 a-2}{6 a}\right) C_{2} \mathrm{~L}^{2}=0$

Solving Equations (14) and (15) simultaneously by substitution method;

$$
\begin{aligned}
& C_{2}=\left(\frac{72 a^{3}-96 a^{2}+30 a}{12 a^{3}-18 a^{2}+8 a-1}\right) \frac{F L}{E W H^{3}} \\
& C_{3}=\frac{12 a(1-a)}{\left(6 a^{2}-6 a+1\right)} \cdot \frac{F}{E W H^{3}}
\end{aligned}
$$

Substituting the values of $\mathrm{C}_{2}$ and $\mathrm{C}_{3}$ back into Equation (7); we have:

$$
y(x)=\frac{F L x^{2}}{E W H^{3}}\left[\left(\frac{72 a^{3}-96 a^{2}+30 a}{12 a^{3}-18 a^{2}+8 a-1}\right)+\frac{12 a(1-a) x}{\left(6 a^{2}-6 a+1\right) L}\right]
$$

For tip deflection of the cantilever beam, $x=L$, substituting into Equation (18), we have;

$$
y(L)=\left(\frac{288 a^{5}-648 a^{4}+516 a^{3}-168 a^{2}+18 a}{72 a^{5}-180 a^{4}+168 a^{3}-72 a^{2}+14 a-1}\right) \frac{F L^{3}}{E W H^{3}}
$$

The tapered width of the cantilever beam was then taken as a function of both the width at the fixed end, $W_{1}$ and the width at the free end, $W_{2}$ of the beam as follows;

$$
w(x)=W_{1}\left(1-\frac{x}{L}\right)+W_{2}\left(\frac{x}{L}\right)
$$

By using the principle of virtual work in order to find the strain energy in the beam, it follows that;

$U=\frac{E H^{3}}{24} \int_{0}^{L}\left[W_{1}\left(1-\frac{x}{L}\right)+W_{2}\left(\frac{x}{L}\right)\right]\left[\frac{d^{2} y}{d x^{2}}\right]^{2} d x-F\left(C_{2} \mathrm{~L}^{2}+\right.$ $\left.C_{3} \mathrm{~L}^{3}\right)$

$U=\frac{E H^{3}}{24} \int_{0}^{L}\left[W_{1}\left(1-\frac{x}{L}\right)+W_{2}\left(\frac{x}{L}\right)\right]\left[2 C_{2}+6 C_{3} x\right]^{2} d x-$ $F\left(C_{2} \mathrm{~L}^{2}+C_{3} \mathrm{~L}^{3}\right)$

By integrating Equation (22) by parts, it follows that

$$
\begin{aligned}
& U=\frac{E H^{3}}{24}\left[C_{3}^{2} L^{3}\left(9 W_{2}+3 W_{1}\right)+C_{2} C_{3} L^{2}\left(8 W_{2}+4 W_{1}\right)+\right. \\
& \left.C_{2}^{2} L\left(2 W_{1}+2 W_{2}\right)\right]-F\left(C_{2} \mathrm{~L}^{2}+C_{3} \mathrm{~L}^{3}\right)
\end{aligned}
$$

In order to find the values of $C_{2}$ and $C_{3}$ that bring the strain energy in Equation (23) to zero, the partial derivatives of $U$ with respect to $C_{2}$ and $C_{3}$ were set to zero and simplified to give Equations (24) and (25) respectively; 
$\frac{E H^{3}}{24}\left[4 C_{2} L\left(W_{1}+W_{2}\right)+4 C_{3} L^{2}\left(W_{1}+2 W_{2}\right)\right]-F L^{2}=0$

$\frac{E H^{3}}{24}\left[4 C_{2} L^{2}\left(W_{1}+2 W_{2}\right)+6 C_{3} L^{3}\left(W_{1}+3 W_{2}\right)\right]-F L^{3}=0$

Solving equations (24) and (25) simultaneously by substitution method yields;

$$
\begin{gathered}
C_{2}=\frac{6 F L}{E H^{3}}\left(\frac{W_{1}^{2}+5 W_{2}^{2}+6 W_{1} W_{2}}{W_{1}^{3}+W_{2}^{3}+5 W_{1} W_{2}^{2}+5 W_{1}^{2} W_{2}}\right) \\
C_{3}=\frac{-12 W_{2}}{\left(4 W_{1} W_{2}+W_{1}^{2}+W_{2}^{2}\right)} \cdot \frac{F}{E H^{3}}
\end{gathered}
$$

The Equation of the deflection curve of the cantilever beam becomes;

$$
\begin{aligned}
& y(x)=\frac{6 F L}{E H^{3}}\left[\left(\frac{W_{1}^{2}+5 W_{2}^{2}+6 W_{1} W_{2}}{W_{1}^{3}+W_{2}^{3}+5 W_{1} W_{2}^{2}+5 W_{1}^{2} W_{2}}\right) x^{2}-\right. \\
& \left.\frac{2 W_{2}}{\left(4 W_{1} W_{2}+W_{1}^{2}+W_{2}^{2}\right)} \cdot \frac{x^{3}}{L}\right]
\end{aligned}
$$

For tip deflection of the cantilever beam, $x=L$, substituting this value into Equation (28), we have;

$$
\begin{aligned}
& y(L)=\frac{6 F L}{E H^{3}}\left[\left(\frac{W_{1}^{2}+5 W_{2}^{2}+6 W_{1} W_{2}}{W_{1}^{3}+W_{2}^{3}+5 W_{1} W_{2}^{2}+5 W_{1}^{2} W_{2}}\right) L^{2}-\right. \\
& \left.\frac{2 W_{2}}{\left(4 W_{1} W_{2}+W_{1}^{2}+W_{2}^{2}\right)} \cdot L^{2}\right] \\
& \boldsymbol{y}(L)=\frac{\mathbf{6 F L ^ { 3 }}}{\boldsymbol{E} \boldsymbol{H}^{3}}\left[\left(\frac{\boldsymbol{W}_{1}^{2}+5 \boldsymbol{W}_{2}^{2}+6 W_{1} W_{2}}{\boldsymbol{W}_{1}^{3}+\boldsymbol{W}_{2}^{3}+5 \boldsymbol{W}_{1} \boldsymbol{W}_{2}^{2}+5 \boldsymbol{W}_{1}^{2} \boldsymbol{W}_{2}}\right)-\right. \\
& \left.\frac{2 \boldsymbol{W}_{2}}{\left(4 \boldsymbol{W}_{1} \boldsymbol{W}_{\mathbf{2}}+\boldsymbol{W}_{\mathbf{1}}^{2}+\boldsymbol{W}_{2}^{2}\right)}\right]
\end{aligned}
$$

\section{CANTILEVER BEAM WITH A TAPERED HEIGHT AND CONSTANT WIDTH}

The width of the cantilever beam was then kept constant and the height tapered. First, the tapered height was taken as a function of only the height at the fixed end, $\mathrm{H}$ of the cantilever beam without including the height at the free end as shown in Equation (31).

$$
h(x)=H\left(1-\frac{x}{b L}\right)
$$

Where $\frac{1}{b}=$ percentage taper of the cantilever height,

Again, by applying the principle of virtual work, it follows that:

$$
\mathrm{W}_{\text {bend }}=\frac{1}{2} \mathrm{E} \int_{0}^{\mathrm{L}} \mathrm{I}_{\mathrm{z}}(\mathrm{x})\left[\frac{\mathrm{d}^{2} \mathrm{y}}{\mathrm{dx}^{2}}\right]^{2} \mathrm{dx}
$$

The moment of inertia of the beam with tapered height and constant width is as follows;

$$
I_{z}(x)=\frac{W[h(x)]^{3}}{12}
$$

Where $h(x)$ is the height at a distance $x$ from the fixed end of the cantilever beam

Therefore, the strain energy becomes;

$$
\begin{aligned}
& U=\frac{1}{2} E \int_{0}^{L} \frac{W[h(x)]^{3}}{12}\left[\frac{d^{2} y}{d x^{2}}\right]^{2} d x-F\left(C_{2} \mathrm{~L}^{2}+C_{3} \mathrm{~L}^{3}\right) \\
& U=\frac{E W H^{3}}{24} \int_{0}^{L}\left(1-\frac{x}{b L_{c}}\right)^{3}\left[\frac{d^{2} y}{d x^{2}}\right]^{2} d x-F\left(C_{2} \mathrm{~L}^{2}+C_{3} \mathrm{~L}^{3}\right) \\
& U=\frac{E W H^{3}}{24} \int_{0}^{L}\left(1-\frac{x}{b L_{c}}\right)^{3}\left[2 C_{2}+6 C_{3} x\right]^{2} d x-F\left(C_{2} \mathrm{~L}^{2}+\right. \\
& \left.C_{3} \mathrm{~L}^{3}\right)
\end{aligned}
$$

By using the integration by parts formula to solve Equation (36), we have:

$$
\int u d v=u v-\int v d u
$$

Where $u=\left(1-\frac{x}{b L_{c}}\right)^{3}$ and $\mathrm{dv}=\left[2 C_{2}+6 C_{3} x\right]^{2} \mathrm{dx}$

$U=\frac{E W H^{3}}{24}\left[4 C_{2}^{2} L\left(1-\frac{3}{2 b}+\frac{1}{b^{2}}-\frac{1}{4 b^{3}}\right)+12 C_{2} C_{3} L^{2}(1-\right.$

$\left.\left.\frac{2}{b}+\frac{3}{2 b^{2}}-\frac{2}{5 b^{3}}\right)+12 C_{3}^{2} L^{3}\left(1-\frac{9}{4 b}+\frac{9}{5 b^{2}}-\frac{1}{2 b^{3}}\right)\right]-$

$F\left(C_{2} \mathrm{~L}^{2}+C_{3} \mathrm{~L}^{3}\right)$

In order to find the values of $C_{2}$ and $C_{3}$ that brings the strain energy in Equation (38) to zero, the partial derivatives of $U$ with respect to $C_{2}$ and $C_{3}$ will be set to zero

$$
\begin{aligned}
& \frac{\partial U}{\partial C_{2}}=\frac{E W H^{3}}{24}\left[8 C_{2} L\left(1-\frac{3}{2 b}+\frac{1}{b^{2}}-\frac{1}{4 b^{3}}\right)+12 C_{3} L^{2}\left(1-\frac{2}{b}+\right.\right. \\
& \left.\left.\frac{3}{2 b^{2}}-\frac{2}{5 b^{3}}\right)\right]-F L^{2}=0 \\
& \frac{\partial U}{\partial C_{3}}=\frac{E W H^{3}}{24}\left[12 C_{2} L^{2}\left(1-\frac{2}{b}+\frac{3}{2 b^{2}}-\frac{2}{5 b^{3}}\right)+24 C_{3} L^{3}(1-\right. \\
& \left.\left.\frac{9}{4 b}+\frac{9}{5 b^{2}}-\frac{1}{2 b^{3}}\right)\right]-F \mathrm{~L}^{3}=0
\end{aligned}
$$

Solving Equations (39) and (40) simultaneously, we have;

$$
\begin{aligned}
& C_{2}=\frac{30 F L}{E W H^{3}}\left(\frac{10 b^{6}-25 b^{5}+21 b^{4}-6 b^{3}}{50 b^{6}-150 b^{5}+185 b^{4}-120 b^{3}+45 b^{2}-10 b+1}\right) \\
& C_{3}=\frac{-F}{E W H^{3}}\left(\frac{100 b^{6}-300 b^{5}+250 b^{4}-70 b^{3}}{50 b^{6}-150 b^{5}+185 b^{4}-120 b^{3}+45 b^{2}-10 b+1}\right)
\end{aligned}
$$


Substituting the values of $C_{2}$ and $C_{3}$ back into the deflection curve equation (Equation 7) of the beam;

$y(x)=\frac{30 F L x^{2}}{E W H^{3}}\left(\frac{10 b^{6}-25 b^{5}+21 b^{4}-6 b^{3}}{50 b^{6}-150 b^{5}+185 b^{4}-120 b^{3}+45 b^{2}-10 b+1}\right)-$

$\frac{F x^{3}}{E W H^{3}}\left(\frac{100 b^{6}-300 b^{5}+250 b^{4}-70 b^{3}}{50 b^{6}-150 b^{5}+185 b^{4}-120 b^{3}+45 b^{2}-10 b+1}\right)$

$y(x)=$

$\frac{F}{E W H^{3}}\left[\frac{30 L\left(10 b^{6}-25 b^{5}+21 b^{4}-6 b^{3}\right) x^{2}-\left(100 b^{6}-300 b^{5}+250 b^{4}-70 b^{3}\right) x^{3}}{50 b^{6}-150 b^{5}+185 b^{4}-120 b^{3}+45 b^{2}-10 b+1}\right]$

For tip deflection of the cantilever beam, $\mathrm{x}=\mathrm{L}$, substituting into Equation (44), we have;

$y(L)=\frac{F L^{3}}{E W H^{3}}\left(\frac{200 b^{6}-450 b^{5}+380 b^{4}-110 b^{3}}{50 b^{6}-150 b^{5}+185 b^{4}-120 b^{3}+45 b^{2}-10 b+1}\right)$

The tapered height of the cantilever beam was then taken as a function of both the height at the fixed end, $h_{1}$ and the height at the free end, $h_{2}$ of the beam as follows;

$h(x)=h_{1}\left(1-\frac{x}{L}\right)+h_{2}\left(\frac{x}{L}\right)$

By using the principle of virtual work in order to find the strain energy in the beam, it follows that;

$U=\frac{E W}{24} \int_{0}^{L}\left[h_{1}\left(1-\frac{x}{L}\right)+h_{2}\left(\frac{x}{L}\right)\right]^{3}\left[\frac{d^{2} y}{d x^{2}}\right]^{2} d x-F\left(C_{2} \mathrm{~L}^{2}+\right.$

$\left.C_{3} \mathrm{~L}^{3}\right)$

$U=\frac{E W}{24} \int_{0}^{L}\left[h_{1}\left(1-\frac{x}{L}\right)+h_{2}\left(\frac{x}{L}\right)\right]^{3}\left[2 C_{2}+6 C_{3} x\right]^{2} d x-$

$F\left(C_{2} \mathrm{~L}^{2}+C_{3} \mathrm{~L}^{3}\right)$

By integrating Equation (48) by parts, it follows that;

$U=\frac{E W}{24}\left[C_{2}^{2} L\left(h_{1}^{3}+h_{2}^{3}+h_{1} h_{2}^{2}+h_{1}^{2} h_{2}\right)+\frac{6 C_{2} C_{3} L^{2}}{5}\left(h_{1}^{3}+\right.\right.$

$\left.4 h_{2}^{3}+3 h_{1} h_{2}^{2}+2 h_{1}^{2} h_{2}\right)+\frac{3}{5} C_{3}^{2} L^{3}\left(h_{1}^{3}+10 h_{2}^{3}+6 h_{1} h_{2}^{2}+\right.$

$\left.\left.3 h_{1}^{2} h_{2}\right)\right]-F\left(C_{2} \mathrm{~L}^{2}+C_{3} \mathrm{~L}^{3}\right)$

In order to find the values of $C_{2}$ and $C_{3}$ that bring the strain energy in Equation (49) to zero, the partial derivatives of $U$ with respect to $C_{2}$ and $C_{3}$ were set to zero and simplified to give Equations (50) and (51) respectively;

$$
\begin{aligned}
& \frac{E W}{24}\left[2 C_{2} L\left(h_{1}^{3}+h_{2}^{3}+h_{1} h_{2}^{2}+h_{1}^{2} h_{2}\right)+\frac{6 C_{3} L^{2}}{5}\left(h_{1}^{3}+4 h_{2}^{3}+\right.\right. \\
& \left.\left.3 h_{1} h_{2}^{2}+2 h_{1}^{2} h_{2}\right)\right]-F L^{2}=0
\end{aligned}
$$

$$
\begin{aligned}
& \frac{E W}{24}\left[\frac{6 C_{2} L^{2}}{5}\left(h_{1}^{3}+4 h_{2}^{3}+3 h_{1} h_{2}^{2}+2 h_{1}^{2} h_{2}\right)+\frac{6}{5} C_{3} L^{3}\left(h_{1}^{3}+\right.\right. \\
& \left.\left.10 h_{2}^{3}+6 h_{1} h_{2}^{2}+3 h_{1}^{2} h_{2}\right)\right]-F L^{3}=0
\end{aligned}
$$

Solving Equations (50) and (51) simultaneously by substitution method yields;

$$
\begin{aligned}
& C_{2}=\frac{30 F L}{E W}\left(\frac{6 h_{2}^{3}+3 h_{1} h_{2}^{2}+h_{1}^{2} h_{2}}{h_{1}^{6}+h_{2}^{6}+4 h_{1}^{5} h_{2}+10 h_{1}^{4} h_{2}^{2}+20 h_{1}^{3} h_{2}^{3}+10 h_{1}^{2} h_{2}^{4}+4 h_{1} h_{2}^{5}}\right) \\
& C_{3}=\frac{10 F}{E W}\left(\frac{2 h_{1}^{3}-7 h_{2}^{3}-4 h_{1} h_{2}^{2}-h_{1}^{2} h_{2}}{h_{1}^{6}+h_{2}^{6}+4 h_{1}^{5} h_{2}+10 h_{1}^{4} h_{2}^{2}+20 h_{1}^{3} h_{2}^{3}+10 h_{1}^{2} h_{2}^{4}+4 h_{1} h_{2}^{5}}\right)
\end{aligned}
$$

The equation of the deflection curve of the cantilever beam becomes;

$y(x)=\frac{10 F}{E W}\left(\frac{3 L\left(6 h_{2}^{3}+3 h_{1} h_{2}^{2}+h_{1}^{2} h_{2}\right) x^{2}+\left(2 h_{1}^{3}-7 h_{2}^{3}-4 h_{1} h_{2}^{2}-h_{1}^{2} h_{2}\right) x^{3}}{h_{1}^{6}+h_{2}^{6}+4 h_{1}^{5} h_{2}+10 h_{1}^{4} h_{2}^{2}+20 h_{1}^{3} h_{2}^{3}+10 h_{1}^{2} h_{2}^{4}+4 h_{1} h_{2}^{5}}\right)$

For tip deflection of the cantilever beam, $x=L$, substituting into Equation (54), we have:

$$
\begin{aligned}
& y(L)= \\
& \frac{10 F L^{3}}{E W}\left(\frac{2 h_{1}^{3}+11 h_{2}^{3}+5 h_{1} h_{2}^{2}+2 h_{1}^{2} h_{2}}{h_{1}^{6}+h_{2}^{6}+4 h_{1}^{5} h_{2}+10 h_{1}^{4} h_{2}^{2}+20 h_{1}^{3} h_{2}^{3}+10 h_{1}^{2} h_{2}^{4}+4 h_{1} h_{2}^{5}}\right)
\end{aligned}
$$

\section{DETERMINISTIC OPTIMISATION}

The deterministic optimisation of the cantilever beam with a tapered width and constant height was done. It was optimised for the geometrical properties that gave the minimum displacement at the tip with the height and length of the cantilever kept constant. The formulation is as follows;

Find the values of $W_{1}$ and $W_{2}$ which minimises the objective function shown below;

Minimise deflection at the tip, $y(\mathrm{~L})=$ $\frac{6 F L^{3}}{E H^{3}}\left[\left(\frac{W_{1}^{2}+5 W_{2}^{2}+6 W_{1} W_{2}}{W_{1}^{3}+W_{2}^{3}+5 W_{1} W_{2}^{2}+5 W_{1}^{2} W_{2}}\right)-\frac{2 W_{2}}{\left(4 W_{1} W_{2}+W_{1}^{2}+W_{2}^{2}\right)}\right]$

Constant volume constraint: $\quad \frac{W_{1}+W_{2}}{2} \times H \times L$

The design variables for deflection minimisation of the cantilever beam design are shown in Table 2. The applied force at the tip of the tapered cantilever beam was taken as $4.75 \mathrm{KN}$ while the elastic modulus of the material, $\mathrm{E}$ was taken as $205,000 \mathrm{KN} / \mathrm{m}^{2}$. The height and length of the cantilever beam were taken as $0.45 \mathrm{~m}$ and $2.5 \mathrm{~m}$ respectively. The ModelCenter software was used for the deterministic optimisation as follows. The formula for calculating the volume of 
the beam was linked with the governing end deflection formula in an excel file. The excel file was then embedded in an optimisation loop. An alternative way of doing this is by creating a script file for the volume and linking it with the deflection formula in the excel file, and embedding both files in an optimisation loop. The constant volume constraint is applied by keeping the same value of volume in the upper bound as in the lower bound. This was done by keeping an upper bound limit of the volume in order not to overconstrain the optimisation problem. The Darwin algorithm was used for the optimisation as the Dot Sequential Quadratic Programming algorithm was not appropriate. The OptLib gradient optimiser and the Design Explorer were also very suitable for the optimisation problem. It took too long for the optimisation problem to converge when the sequential quadratic programming algorithm was employed, therefore leading to an infeasible design. The OptLib gradient optimiser and Design Explorer were very suitable in as they gave more economical designs as compared to the Darwin algorithm. They are also less computationally expensive as compared to the Darwin algorithm. The dialogue box for the deterministic optimisation in ModelCenter is shown in Figure 2.

\section{RESULTS AND DISCUSSION}

The validation of the structural systems (e.g. cantilever beam) was done by the finite element method, with mesh densities, element types, and boundary conditions adding to the epistemic uncertainties. The choice of the element type, shape and geometry, boundary conditions and constraints played major roles in the performance of the model.
The Abaqus finite element analysis software was used for this purpose. Figure 3 shows a model of the tapered width, constant height cantilever beam in Abaqus software. Different kinds of brick finite elements were used in the verification of the analytical solutions obtained.

The validation of the analytical solution in Abaqus finite element software shows that the best finite elements for the analysis of the tapered width, constant height cantilever beam are the 20-nodes quadratic brick elements with reduced integration (see Figure 4). The triangular prism element type and the quadratic tetrahedron element were not suitable for this analysis as they did not give consistent displacement values at the nodes. It can be seen from the bar chart that the mesh with the 20-nodes brick elements gives end deflection values which are very close to $0.05055 \mathrm{~m}$. More specifically C3D20RH (A 20node quadratic brick, a hybrid element with linear pressure and reduced integration) gave an end displacement of $0.05035 \mathrm{~m}$.

Since this is the closest approximation to the actual end deflection of $0.05352 \mathrm{~m}$, this element was therefore adopted for mesh refinement analysis. This difference between the actual deflection value and the deflection value predicted by the C3D20RH constitutes an epistemic uncertainty.

Table 2: Design values for the tapered cantilever

\begin{tabular}{llll}
\multicolumn{4}{c}{ beam with constant height } \\
\hline Design & Start & Lower & Upper \\
Variable & value & limit & limit \\
\hline $\mathrm{W}_{1}(\mathrm{~m})$ & 0.30 & 0.10 & 0.50 \\
$\mathrm{~W}_{2}(\mathrm{~m})$ & 0.175 & 0.10 & 0.50 \\
\hline
\end{tabular}

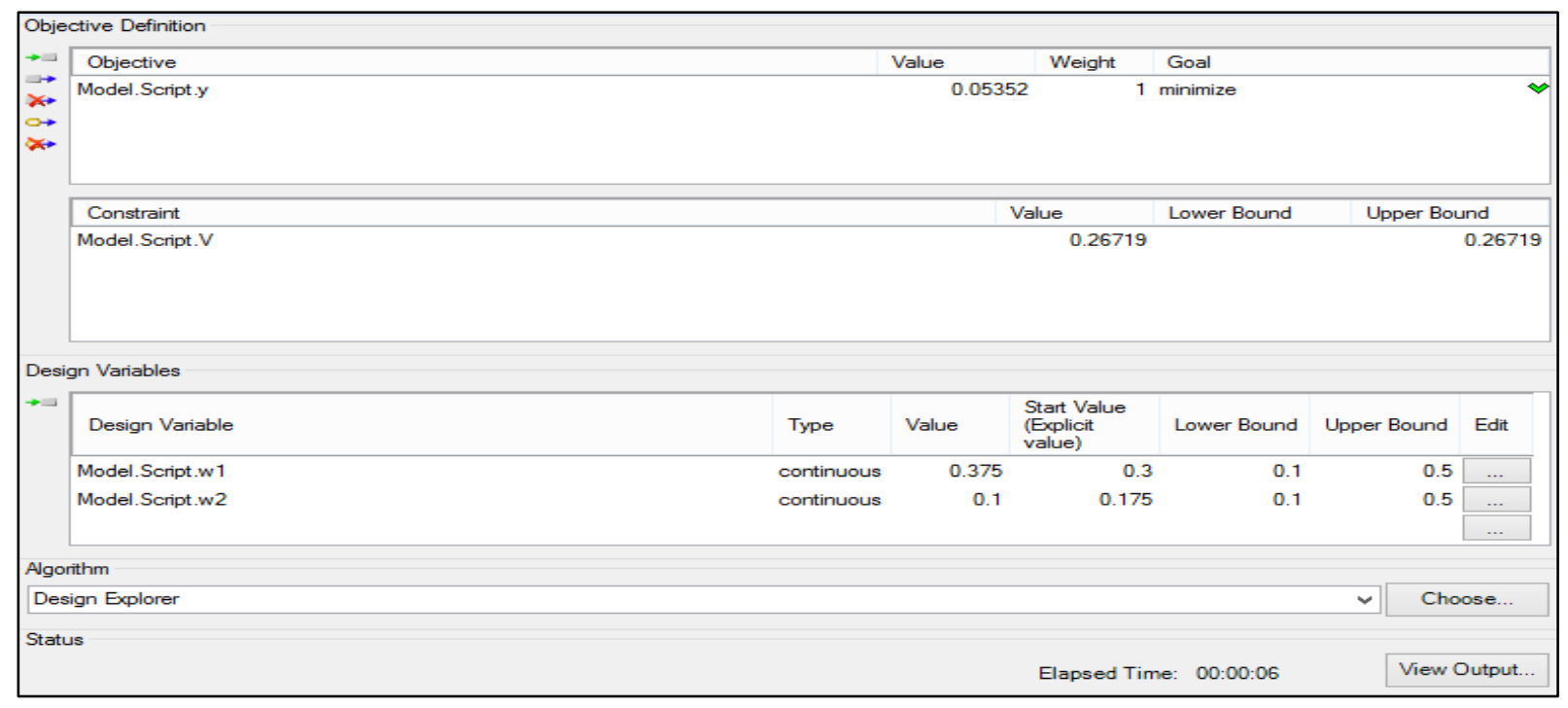

Figure 2: The optimisation tool dialogue box 
The deterministic optimisation of the tapered width, constant height cantilever beam for the minimum deflection at the tip produced a lower value of 0.05352 $\mathrm{m}$ for the tip deflection than the initial design $(0.05948$ $\mathrm{m}$ ) as expected while the width at the fixed end of the cantilever increased from $0.30 \mathrm{~m}$ to $0.375 \mathrm{~m}$ and the width at the free end decreased from $0.175 \mathrm{~m}$ to the very minimum of its range, $0.1 \mathrm{~m}$ as shown in Figure 5. This lower deflection value was obtained when these values of the fixed and free ends width were substituted into the governing deflection formula. This affirms that the values gotten are in order.

The increase in the width at the fixed end, w1 of the cantilever beam and the decrease in the length at the free of the deflection optimisation also points to the fact that the end deflection is more sensitive to $\mathrm{w} 1$ than w2. This was confirmed in a sensitive result of a design of experiment using the full factorial method as shown in Figure 6 . It can be seen that $w 1$ has a sensitivity summary of 0.845 while w2 has 0.336 .

The deterministic optimisation by the gradient optimiser, design explorer and Darwin algorithm all gave different forms of convergence history diagrams. Figure 7 and Figure 8 show the convergence history of the optimisation by using the design explorer algorithm and Darwin algorithm respectively. It can be clearly seen from the two convergence histories that since the design explorer is an optimiser that works based on gradient methods, it seeks for the optimum solution by creating series of sloping straight lines.

The carpet plot showing the constant volume constraint and the valid design space is as shown in Figure 9. The blue line represents the volume constraint. The dashed side of the constraint line points to the invalid region of the design space. It can be seen that for a tapered beam of constant height and maximum volume, the minimum deflection will be when $\mathrm{w}_{2}$ is at its lower bound and $\mathrm{w}_{1}$ is as large as it can be subject to the volume constraint or its upper bound; or when w1 is at its upper bound and $\mathrm{w} 2$ is as large as it can be subject to the volume constraint or its upper bound. In this case, the width of the cantilever beam at the free end decreased to its lower limit, $0.1 \mathrm{~m}$ and the width at the fixed end increased from $0.3 \mathrm{~m}$ to $0.375 \mathrm{~m}$. This may be due to the high bending moments at the fixed end of a cantilever beam as a result of the applied point load at the free end. The increase in the width at the fixed end is for a higher section to resist the applied moments.

The response surface plot for the deflection of the tapered width, constant height cantilever beam is as shown in Figure 10. The response surface seems to be or approximated to a second order model. A detailed look at the response surface also shows that the minimum displacement of $0.05352 \mathrm{~m}$ occurs at the point where $\mathrm{w} 1$ is equal to $0.375 \mathrm{~m}$ and $\mathrm{w} 2$ is equal to $0.1 \mathrm{~m}$.

The scatter plot also reveals a strong correlation between the end displacement and the elastic modulus of the beam material. It can be seen in Figure 11 that as the elastic modulus of the material increases the deflection decreases, which is an affirmation of the relationship between deflection and $E$ as seen in Equation 30. The deflection of the beam is inversely proportional to the elastic modulus. Also, most of the Monte Carlo samples lie below the limit state $(0.08635$ $\mathrm{m})$. There are just about two cases where the limit state was exceeded for which the elastic modulus goes below $119,277 \mathrm{kN} / \mathrm{m}^{2}$. Therefore the value of the elastic modulus corresponding to the limit state is about $119,277 \mathrm{kN} / \mathrm{m}^{2}$, below which the structure is considered to have failed. This explains why after the probabilistic analysis to determine the limit state, a lower value of $E$ is returned in the data explorer window. A design of this structure at this limit state is actually very robust as only about two samples from the scatter plot violate this limit.

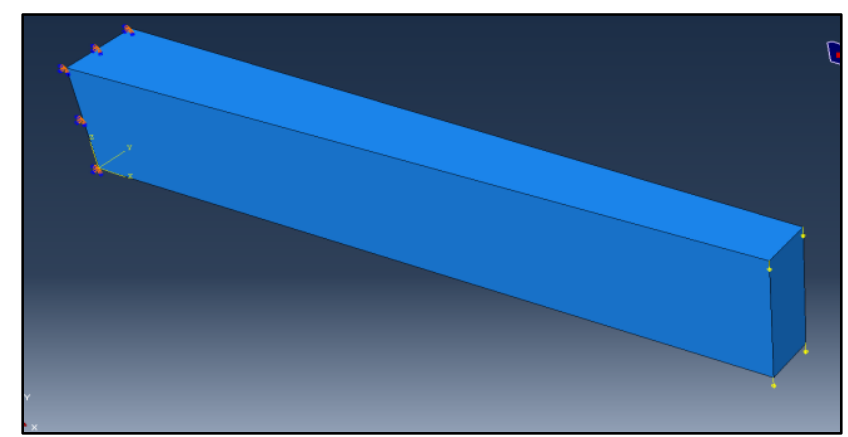

Figure 3: A model of the tapered width, constant height cantilever beam in Abaqus software 


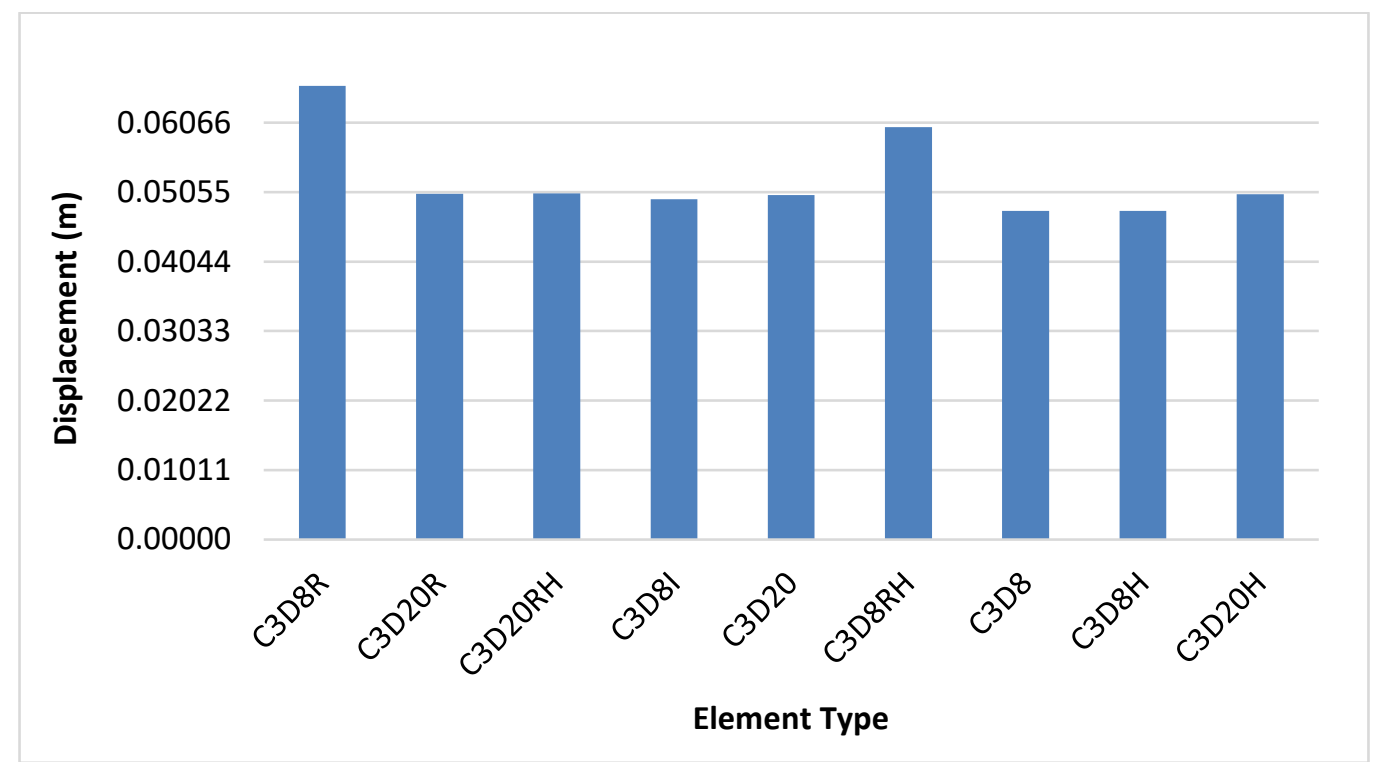

Figure 4: The best element type for the cantilever beams analysis

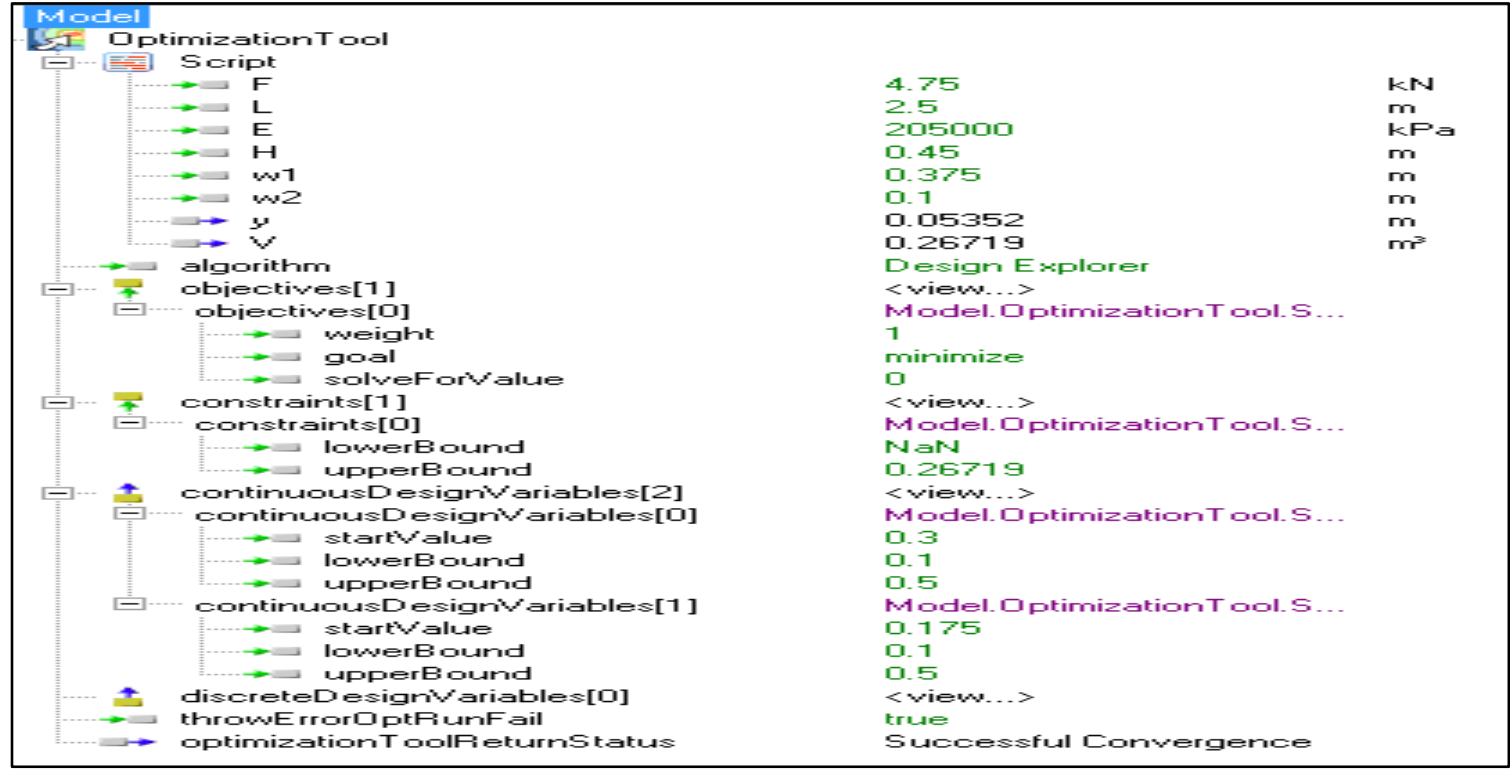

Figure 5: The optimum values for the tapered width, constant height cantilever

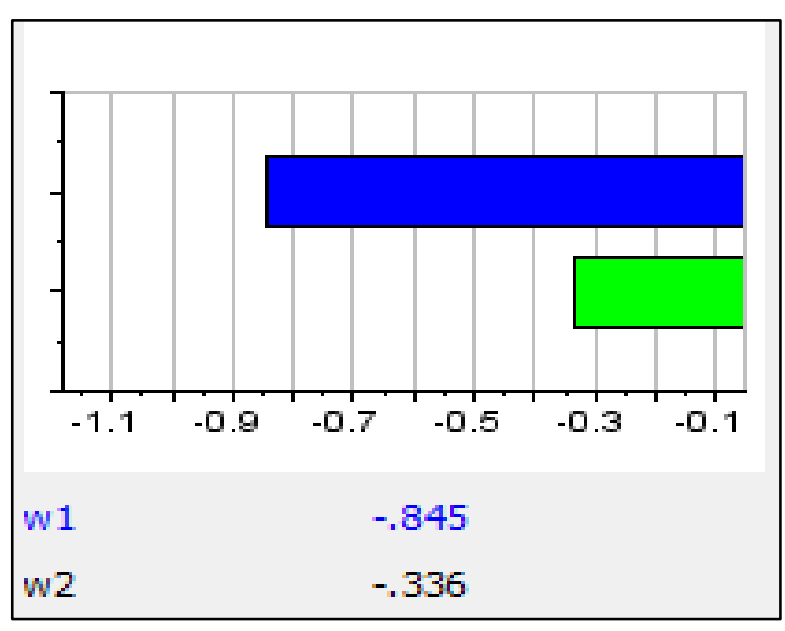

Figure 6: Sensitivity summary

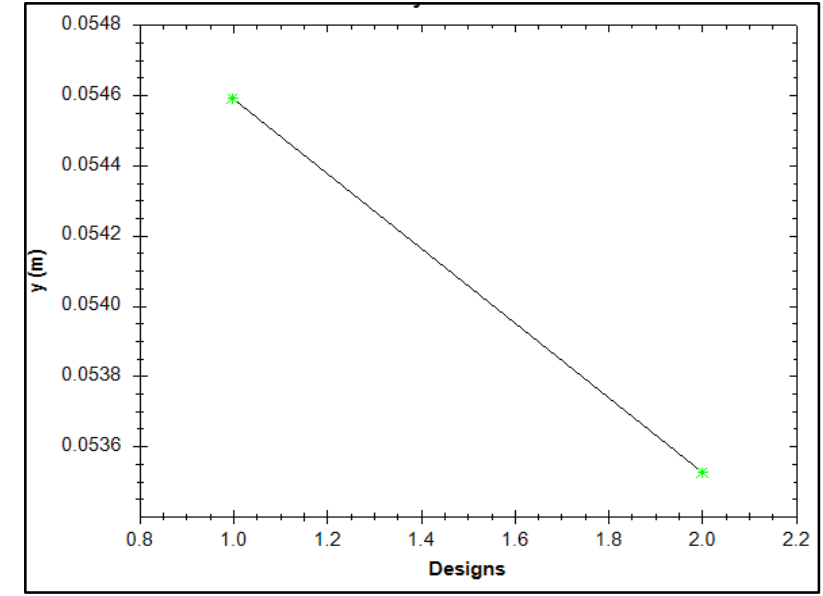

Figure 7: The convergence history by the design explorer algorithm 


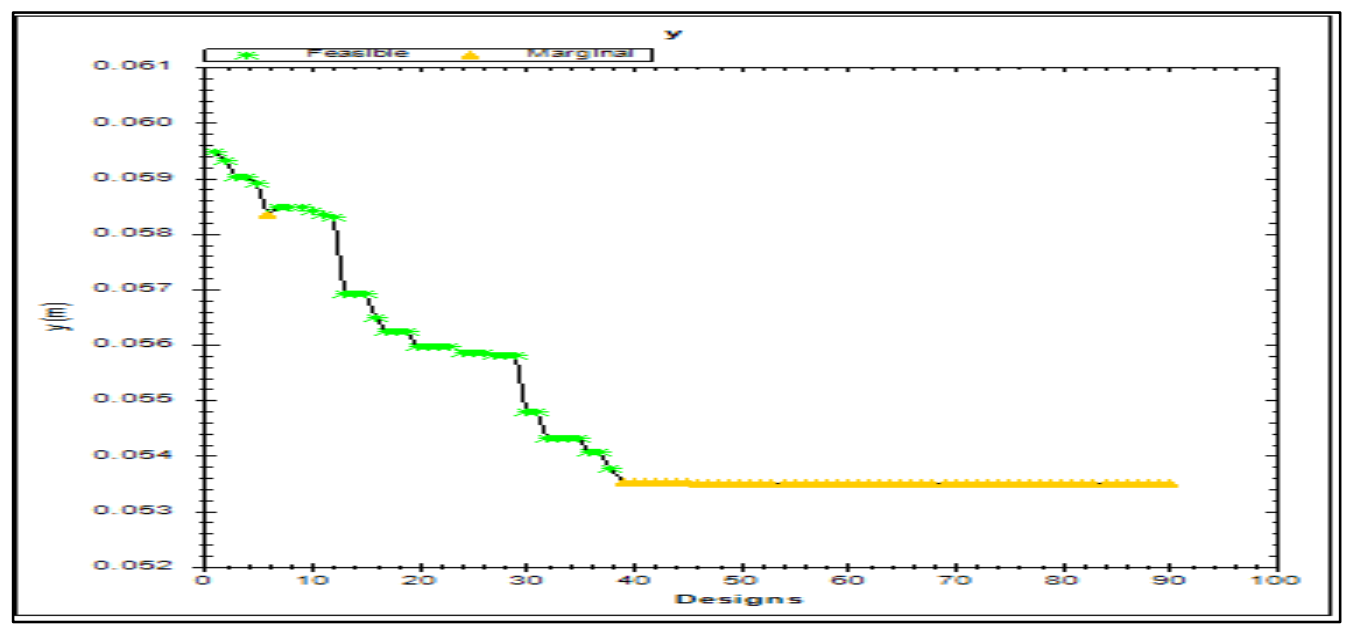

Figure 8: The convergence history by the Darwin algorithm

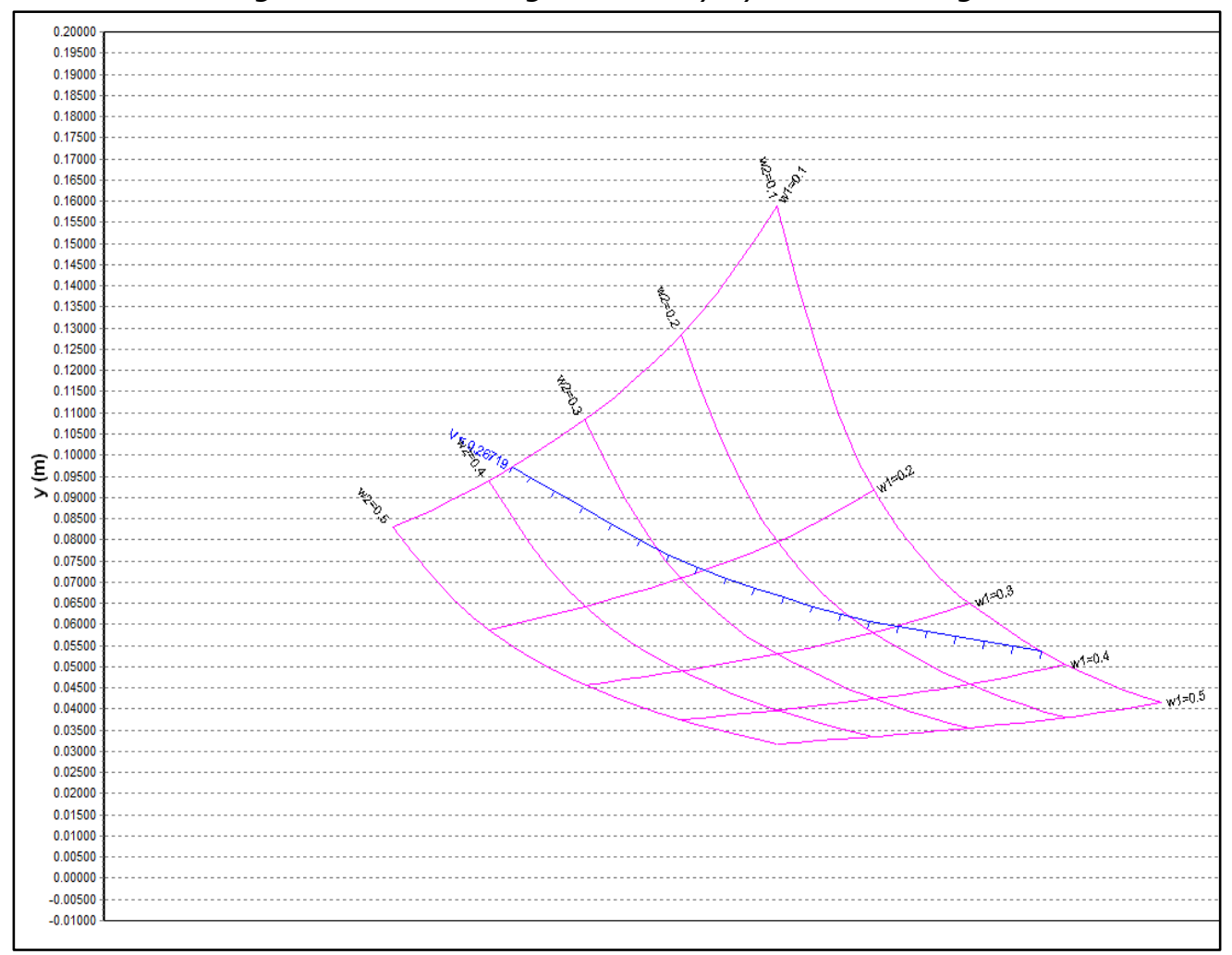

Figure 9: The carpet plot showing the volume constraint and the valid design space

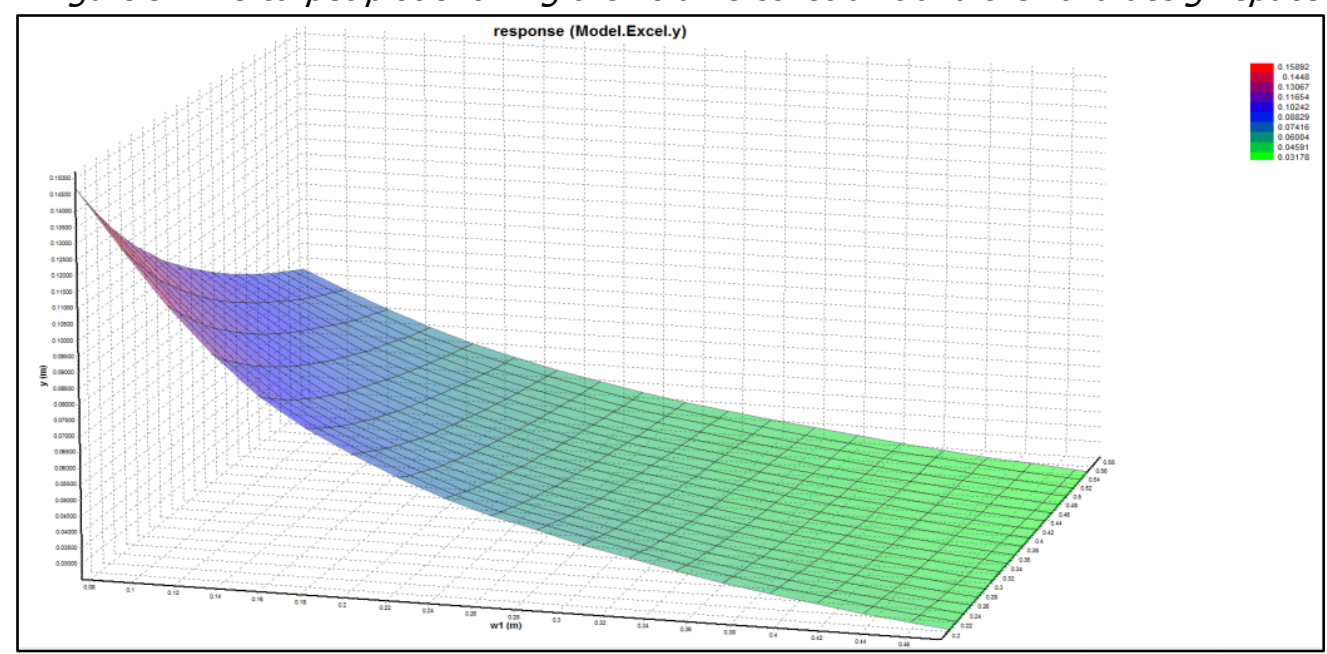

Figure 10: The response surface for the deflection of the tapered width, constant height beam 


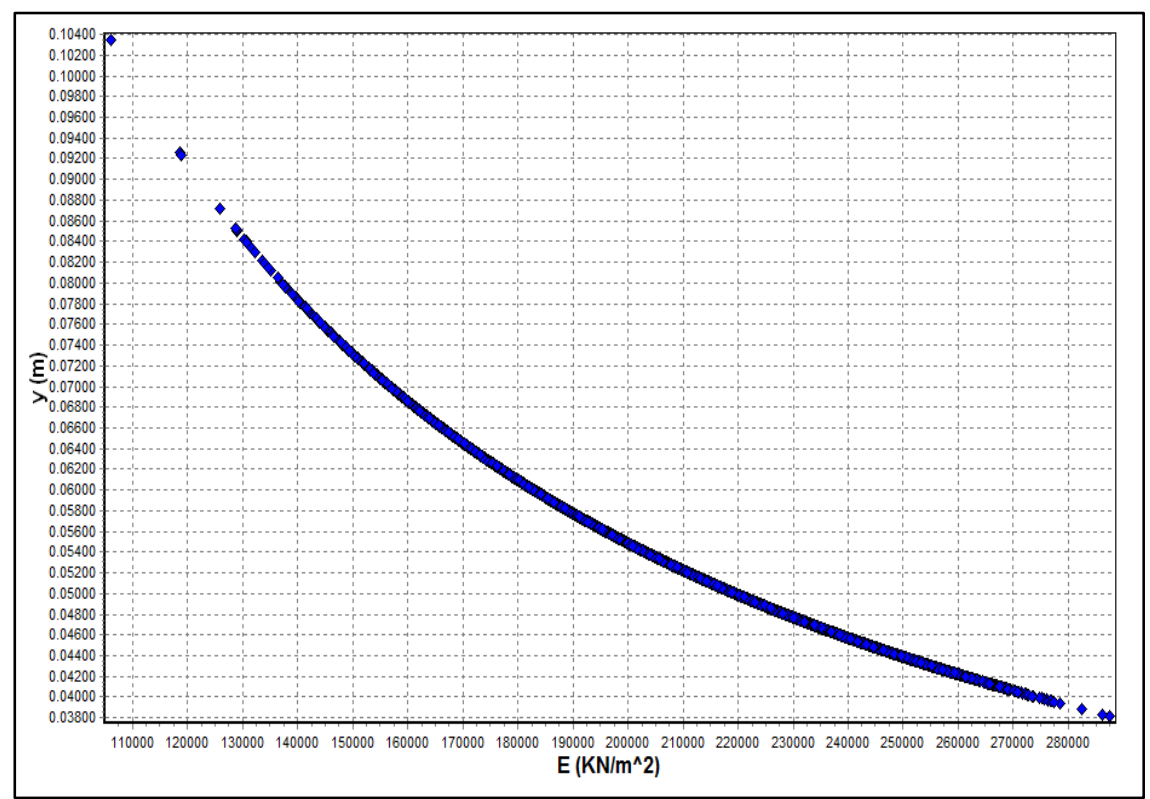

Figure 11: The correlation between the elastic modulus and the end displacement

\section{CONCLUSION}

This work set out to develop and validate deflection equations for tapered cantilever beam. Analytical solutions for the end deflection of various configurations of three-dimensional tapered cantilever beam were developed by using the shape functions of a bar finite element. The validation of these analytical solutions in Abaqus finite element software was done and the C3D20HR brick element was chosen as the best element. It predicted an end displacement to be $0.05038 \mathrm{~m}$ for the tapered width, constant height cantilever beam. This was seen to be an epistemic uncertainty since the prediction was not exact as the real displacement.

The design explorer algorithm was adopted for the deterministic optimisation of the structural systems.

The Darwin algorithm and other evolutionary algorithms were highly computationally expensive, and therefore wasteful for these analyses. The constant volume constraint limited the design space, and as such put a limit on the feasible optimum solution.

Future works in this area can be focused on modification of the available optimisation algorithms to suitable robust algorithms for the multiple objective optimisations of specific structural systems with multiple constraints. The roles epistemic and other uncertainties play in the optimisation of structural systems can also be investigated.

\section{REFERENCES}

[1] Khurmi, R.S. (2005). Strength of Materials (Mechanics of Solids). S. Chand \& Company Ltd.

[2] Smith, M. (2009). ABAQUS/Standard User's Manual, Version 6.9. Providence, RI: Dassault Systemes Simulia Corp.

[3] Carmichael, D.G. (1981). Structural Modelling and Optimization. Ellis Horwood Limited

[4] Ben-Tal, A. and Nemirovski, A. (2002) 'Robust optimization-methodology and applications', Mathematical Programming, 92(3), pp. 453-480.

[5] Rao, S., S. (2009) Engineering Optimisation: Theory and Practice. New Jersey: John Wiley \& Sons, Inc.

[6] Belegundu, A.D. (1983) 'Study of mathematical programming methods for structural optimization', Dissertation Abstracts International Part B: Science and Engineering [DISS. ABST. INT. PT. BSCI. \& ENG.], 43(12), p. 1983.

[7] Eschenauer, H.A. and Olhoff, N. (2001) 'Topology optimization of continuum structures: a review', Applied Mechanics Reviews, 54(4), pp. 331-390.

[8] Zienkiewicz, O.C. and Taylor, R.L. (2005) The finite element method for solid and structural mechanics. Butterworth-heinemann.

[9] Moaveni, S. (2008) Finite Element Analysis: Theory and Application with ANSYS. New Jersey: Pearson Education, Inc.

[10] Cook, R.D., Malkus, D.S., Plesha, M.E. and Witt, R.J. (2002) Concepts and Applications of Finite 
Element Analysis. New York: John Wiley \& Sons, Inc.

[11] Gosling, P.D., Bridgens, B.N. and Zhang, L. (2013) 'Adoption of a reliability approach for membrane structure analysis', Structural Safety, 40 , pp. $39-50$

[12] Kinser, D.E. and Moses, F. (1967) 'Optimum structural design with failure probability constraints', AIAA Journal, 5(6), pp. 1152-1158.

[13] White, D.W. and Abel, J.F. (1989) 'Test of Shell Finite Element Accuracy and Robustness', Finite
Elements in Analysis and Design, 6(2), pp. 129151.

[14] Bachmann, A., Kunde, M., Litz, M. and Schreiber, A. (2010) 'Advances in generalization and decoupling of software parts in a scientific simulation workflow system'.

[15] Gurugubelli, S. and Kallepalli, D. (2014) 'Weight and deflection optimization of Cantilever beam using a modified Non-Dominated sorting Genetic Algorithm', IOSR Journal of Engineering, 4(3), pp. 19-23. 